\title{
Evaluation of chilling injury in mangoes using multispectral imaging
}

\begin{abstract}
Commodities originating from tropical and subtropical climes are prone to chilling injury (CI). This injury could affect the quality and marketing potential of mango after harvest. This will later affect the quality of the produce and subsequent consumer acceptance. In this study, the appearance of CI symptoms in mango was evaluated non-destructively using multispectral imaging. The fruit were stored at $4{ }^{\circ} \mathrm{C}$ to induce $\mathrm{CI}$ and $12{ }^{\circ} \mathrm{C}$ to preserve the quality of the control samples for 4 days before they were taken out and stored at ambient temperature for $24 \mathrm{hr}$. Measurements using multispectral imaging and standard reference methods were conducted before and after storage. The performance of multispectral imaging was compared using standard reference properties including moisture content (MC), total soluble solids (TSS) content, firmness, $\mathrm{pH}$, and color. Least square support vector machine (LS-SVM) combined with principal component analysis (PCA) were used to discriminate CI samples with those of control and before storage, respectively. The statistical results demonstrated significant changes in the reference quality properties of samples before and after storage. The results also revealed that multispectral parameters have a strong correlation with the reference parameters of $\mathrm{L}^{*}, \mathrm{a}^{*}, \mathrm{TSS}$, and MC. The MC and $\mathrm{L}^{*}$ were found to be the best reference parameters in identifying the severity of CI in mangoes. PCA and LS-SVM analysis indicated that the fruit were successfully classified into their categories, that is, before storage, control, and CI. This indicated that the multispectral imaging technique is feasible for detecting $\mathrm{CI}$ in mangoes during postharvest storage and processing.
\end{abstract}

Keyword: Chilling injury; Fruit quality; Mango; Multispectral imaging; Postharvest storage 